\title{
Relay ARQ Strategies for Single Carrier MIMO Broadband Amplify-and-Forward Cooperative Transmission
}

\author{
Zakaria El-Moutaouakkil ${ }^{(1)}$, Tarik Ait-Idir ${ }^{(2,3)}$, Halim Yanikomeroglu ${ }^{(4)}$, and Samir Saoudi ${ }^{(3)}$ \\ ${ }^{(1)}$ Nokia Siemens Networks, Morocco \\ ${ }^{(2)}$ Communications Systems Department, INPT, Madinat Al Irfane, Rabat, Morocco \\ ${ }^{(3)}$ Signal and Communications Department, INSTITUT TELECOM/TELECOM Bretagne, Brest, France \\ ${ }^{(4)}$ Department of Systems and Computer Engineering, Carleton University, Ottawa, Canada \\ Emails: zakaria.elmou@ieee.org, aitidir@ieee.org, halim@sce.carleton.ca, samir.saoudi@telecom-bretagne.eu
}

\begin{abstract}
This paper investigates throughput-efficient relay ARQ protocols for single carrier MIMO systems with amplify-and-forward relaying. We focus on reducing the multiplexing loss due to the half-duplex operation at the relay. We introduce two new relaying protocols where both ARQ and relaying are jointly targeted. Compared to the conventional relaying techniques, the proposed mechanisms require only one time slot duration for transmitting the entire data packet at each ARQ round. We also focus on performance evaluation, and show that the new techniques provide significant improvement in average throughput while maintaining good outage probability performance.
\end{abstract}

Index Terms-Amplify-and-Forward, ARQ, MIMO, cooperative relaying, outage probability, average throughput.

\section{INTRODUCTION}

Recently, the concept of relaying has been recognized as an efficient solution for improving the diversity gain of wireless access systems where a wide radio coverage is required [1], [2]. This concept could bring interesting enhancements to eventually both diversity and multiplexing gains through the exploitation of the so-called cooperative relaying [3], [4]. In cooperative relaying, communication between the source and the destination nodes is performed with the aid of multiple relays acting as packet retransmitters. This technique presents a generalization of the classical automatic repeat request (ARQ) mechanisms. It provides a substantial increase in the diversity gain compared to conventional ARQ, especially in the case of the so-called long-term quasi-static ARQ channels, where the channel connecting the source and destination nodes varies very slowly from round to round. Many research works have then focused on the study of cooperative relaying under many frameworks (see for instance [5]-[9] and references therein). Unfortunately, in cooperative relaying the diversity gain is increased at the expense of throughput loss due to the half-duplex constraint at relay nodes. Different methods have been proposed to recover this loss. In [10], successive relaying using repetition coding has been introduced for a two relay wireless network with flat fading. In [11], relay selection methods have been proposed for cooperative communication with decode-and-forward (DF) relaying.

A prominent alternative to reducing the throughput loss in relayaided transmission mechanisms is the combination of both ARQ and relaying. This approach would significantly reduce the half-duplex multiplexing loss by activating ARQ for rare erroneously decoded data packets, when they occur. Approaches targeting the joint design of ARQ and relaying in one common protocol have recently received more interest (see for instance [12]-[14]). Motivated by the above suggestion, we investigate throughput efficient cooperative transmission techniques where both ARQ and relaying are jointly designed.

In this paper, we focus on single carrier multiple-input-multipleoutput (MIMO) broadband cooperative transmission with amplify-andforward (AF) relaying. Most of the research work that has been carried out in the area of cooperative communications has focused on flat fading channels with single antennas. Relaying techniques for frequency selective fading channels have recently been investigated by some authors (see for instance [9], [15], [16]). We design throughputefficient relaying protocols where the multiplexing loss due to the halfduplex transmission constraint is reduced while providing interesting error performance. We introduce two new relay ARQ protocols where the total time required for transmitting one data packet is significantly reduced compared with conventional relaying methods. We also evaluate both the outage error probability and average throughput performance of the proposed schemes, and show that they outperform classical cooperative relaying.

Throughout the paper, we use the following notation. Superscripts ${ }^{\mathrm{T}}$, and ${ }^{\mathrm{H}}$ denote transpose, and Hermitian transpose, respectively. $\mathbb{E}[$. is the mathematical expectation of the argument (.). When $\mathbf{X}$ is a square matrix, $\operatorname{diag}\{\mathbf{X}\}$ denotes the row vector corresponding to the diagonal of $\mathbf{X} . \mathbf{I}_{N}$ is the $N \times N$ identity matrix, and $\mathbf{0}_{N \times Q}$ denotes an all zero $N \times Q$ matrix. The remainder of the paper is organized as follows: In Section II, we briefly describe the considered framework then introduce the proposed relay ARQ protocols and their corresponding communication models. In Section III, we provide the outage rate of the new schemes and focus on the outage probability and average throughput. Simulation results are provided in Section IV. Finally, the paper is concluded in Section V.

\section{RELAY ARQ SYSTEM}

\section{A. Brief Description of the Concept}

We consider a relay-assisted single carrier communication system where one relay node $R$ assists the communication between a source node $S$ and a destination node $D$. These nodes are equipped with $N_{R}$, $N_{S}$, and $N_{D}$ antennas, respectively. The relay node $R$ is supposed to be either a stationary station or a fixed relay that is not engaged in transmitting or receiving. Also, we assume that relaying works under the framework of half-duplex amplify-and-forward protocol defined over two consecutive time-slots (TS)s. The relay system under consideration uses an ARQ protocol at the upper layer. Packet retransmission is activated upon frame decoding failure at the destination node $D$. It follows the so-called Chase-type ARQ, where both the encoding and the space-time mapping and modulation schemes remain unchanged during all ARQ rounds (i.e., the source node $S$ retransmits the same symbol packet at each ARQ round). At the destination node $D$, ARQ retransmissions are combined until successful packet decoding is achieved. A positive acknowledgment (ACK) is then sent back by node $D$ to $S$ in order to move on to the next data packet. On the contrary, a negative acknowledgment (NACK) is sent back to $S$ in the case of a decoding failure at $D$. The symbol frame is then retransmitted using 


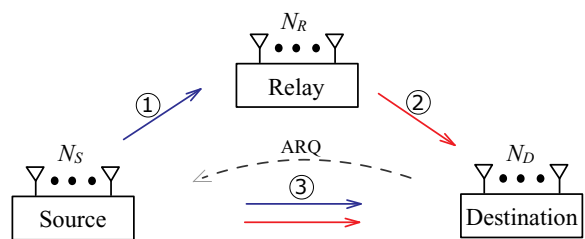

Figure 1. Relay ARQ system model.

two consecutive slots. We say that the system presents an error when the ARQ delay $K$ (index $k=1, \cdots, K$ ), i.e., maximum number of ARQ rounds, is reached and the decoding outcome is still erroneous. The relay ARQ system is depicted in Fig. 1. Since the channel connecting each pair of nodes may experience frequency selective fading, let the number of independent paths in channels 1 (i.e., $S \rightarrow R$ link), 2 (i.e., $R \rightarrow D$ link), and 3 (i.e., $S \rightarrow D$ link) be $L_{S R}, L_{R D}$, and $L_{S D}$, respectively. Therefore, the corresponding quasi-static MIMO channels have matrices $\left\{\mathbf{H}_{l}^{S R^{(k)}}\right\}_{0<l<L_{S R}-1} \in \mathbb{C}^{N_{R} \times N_{S}},\left\{\mathbf{H}_{l}^{R D^{(k)}}\right\}_{0 \leq l<L_{R D}-1} \in$ $\mathbb{C}^{N_{D} \times N_{R}}$, and $\left\{\mathbf{H}_{i, l}^{S D^{(k)}}\right\}_{0 \leq l \leq L_{S D}-1}^{S R} \in \mathbb{C}^{N_{D} \times N_{S}}$ with independent and identically distributed (i.i.d.) entries drawn from zero-mean circularly symmetric complex Gaussian distributions. The channel energies are normalized according to

$$
\left\{\begin{array}{l}
\mathbb{E}\left[\operatorname { d i a g } \left\{\sum_{l=0}^{L_{S R}-1} \mathbf{H}_{l}^{S R^{(k)}} \mathbf{H}_{l}^{\left.\left.S R^{(k)^{\mathrm{H}}}\right\}\right]}=N_{S} \mathbf{I}_{N_{R}},\right.\right. \\
\mathbb{E}\left[\operatorname{diag}\left\{\sum_{l=0}^{L_{R D}-1} \mathbf{H}_{l}^{R D^{(k)}} \mathbf{H}_{l}^{R D^{(k)}} \mathrm{H}\right\}\right]=N_{R} \mathbf{I}_{N_{D}}, \\
\mathbb{E}\left[\operatorname{diag}\left\{\sum_{l=0}^{L_{S D}-1} \mathbf{H}_{i, l}^{S D^{(k)}} \mathbf{H}_{i, l}^{S D^{(k)}}\right\}\right]=N_{D} \mathbf{I}_{N_{D}} .
\end{array}\right.
$$

Note that in the last normalization in (1), $i \in\{1,2\}$ denotes the TS index since the $S \rightarrow D$ link is involved in transmission over two consecutive slots.

\section{B. Relay ARQ Protocol}

Now, let us focus on what happens at ARQ round $k$. Let

$$
\mathbf{x} \triangleq\left[\mathrm{x}_{0}, \ldots, \mathrm{x}_{T-1}\right] \in \mathbb{C}^{N_{S} \times T}
$$

denote the symbol packet at node $S$ generated according to a spacetime bit interleaved coded modulation (STBICM) coding scheme. $T$ (index $t^{\prime}=0, \cdots, T-1$ ) is the total number of channel use (c.u) in $\mathbf{x}$. We assume infinitely deep space-time interleaving and equal power allocation at node $S$, i.e.,

$$
\mathrm{E}\left[\mathrm{x}_{t^{\prime}} \mathrm{x}_{t^{\prime}}^{\mathrm{H}}\right]=\mathbf{I}_{N_{S}}
$$

where $\mathrm{x}_{t^{\prime}}=\left[\mathrm{x}_{t^{\prime}}^{(1)}, \ldots, \mathrm{x}_{t^{\prime}}^{\left(N_{s}\right)}\right]^{\mathrm{T}} \in \mathcal{X}^{N_{S}}$ is the symbol vector transmitted at c.u $t^{\prime}$, and $\mathcal{X}$ is the constellation set. Note that when a NACK is fed back at round $k$, symbol packet $\mathbf{x}$ is entirely retransmitted. The communication protocol is depicted in Fig. 2.a.

First, the source node $S$ proceeds by splitting symbol packet $\mathbf{x}$ into two $N_{S} \times \frac{T}{2}$ sub-packets $\mathbf{z}_{1}=\left[\mathrm{z}_{1,0}, \ldots, \mathrm{z}_{1, T / 2-1}\right]$ and $\mathbf{z}_{2}=$ $\left[\mathrm{z}_{2,0}, \ldots, \mathrm{z}_{2, T / 2-1}\right]$ constructed as

$$
\left\{\begin{array}{ll}
\mathrm{z}_{1, t}=\mathrm{x}_{2 t} & , \quad 0 \leq t \leq \frac{T}{2}-1 \\
\mathrm{z}_{2, t}=\mathrm{x}_{2 t+1} & , \quad 0 \leq t \leq \frac{T}{2}-1
\end{array} .\right.
$$

During the first TS of ARQ round $k$, node $S$ appends a cyclic prefix (CP) symbol word of length $L_{c p}=\max \left\{L_{S R}, L_{R D}, L_{S D}\right\}$ to $\mathbf{z}_{1}$ to avoid inter-block interference (IBI), then broadcasts the resulting symbol packet to both $R$ and $D$ nodes. After removing the CP portion,

\begin{tabular}{|l|c|c|}
\cline { 2 - 3 } \multicolumn{1}{c|}{} & \multicolumn{2}{c|}{ Trans. $(k)$} \\
\cline { 2 - 3 } \multicolumn{1}{c|}{} & $1^{\text {st }}$ TS & $2^{\text {2d }}$ TS \\
\hline$(S)$ & $\mathbf{z}_{1}$ & $\mathbf{z}_{2}$ \\
\hline$(R)$ & $\mathbf{y}_{\mathrm{R}}^{(k)}$ & $\widetilde{\mathbf{y}}_{\mathrm{R}}^{(k)}$ \\
\hline$(D)$ & $\mathbf{y}_{\mathrm{D}}^{1,(k)}$ & $\mathbf{y}_{\mathrm{D}}^{2,(k)}$ \\
\hline
\end{tabular}

(a)

Transmission Period

Figure 2. Relay ARQ Protocol (a), Relay ARQ with slots-mapping reversal (b) for $k=1, \ldots, K$.

the base-band received signal vectors at the level of both relay and destination nodes can be respectively expressed as

$$
\begin{aligned}
\mathbf{y}_{R, t}^{(k)} & =\sqrt{\mathrm{E}_{S R}} \sum_{l=0}^{L_{S R}-1} \mathbf{H}_{l}^{S R^{(k)}} \mathbf{z}_{1,(t-l) \bmod T}+\mathbf{n}_{R, t}^{(k)}, \\
\mathbf{y}_{D, t}^{1,(k)} & =\sqrt{\mathrm{E}_{S D}} \sum_{l=0}^{L_{S D}-1} \mathbf{H}_{1, l}^{S D^{(k)}} \mathbf{z}_{1,(t-l) \bmod T}+\mathbf{n}_{D, t}^{1,(k)},
\end{aligned}
$$

for $t=0, \ldots, T / 2-1$. In (5) and (6), $\mathbf{n}_{\mathrm{R}, t}^{(k)}$ and $\mathbf{n}_{\mathrm{D}, t}^{1,(k)}$ represent the additive spatially and temporally white Gaussian noise vectors at nodes $R$ and $D$ with covariance matrices $N_{0} \mathbf{I}_{N_{R}}$ and $N_{0} \mathbf{I}_{N_{D}}$, respectively. $\mathrm{E}_{S R}$ and $\mathrm{E}_{S D}$ (and similarly $\mathrm{E}_{R D}$ ) are energies capturing the effect of shadowing and path loss in channels 1 and 3, respectively. During the second TS, to ensure unit average transmitted energy per vector entry, the relay node $R$ amplifies the elements of signal vector $\mathbf{y}_{\mathrm{R}, t}^{(k)}$ by the factor $\gamma=1 / \sqrt{N_{S} \mathrm{E}_{S R}+N_{0}}$. The resulting signal block

$$
\widetilde{\mathbf{y}}_{R, t}^{(k)}=\gamma \mathbf{y}_{R, t}^{(k)}, t=1, \ldots, \frac{T}{2}
$$

is transmitted along with the second sub-block $\mathbf{z}_{2}$ from the source node $S$. Therefore, the destination node $D$ will see during the second slot a superposition of two transmitted blocks $\widetilde{\mathbf{y}}_{R, t}^{(k)}$ and $\mathbf{z}_{2}$ after deleting the CP portion of length $L_{c p}$ being added to both of them. The received base-band signal vector at the second TS at the level of node $D$ can therefore be expressed as

$$
\mathbf{y}_{D, t}^{2,(k)}=\sum_{l=0}^{L_{\max }-1} \widetilde{\mathbf{H}}_{l}^{(k)} \mathbf{z}_{(t-l) \bmod T}+\widetilde{\mathbf{n}}_{D, t}^{2,(k)},
$$

where

$$
\left\{\begin{aligned}
\mathbf{z}_{t} & \triangleq\left[\begin{array}{c}
\mathbf{z}_{1, t} \\
\mathbf{z}_{2, t}
\end{array}\right] \in \mathcal{X}^{2 N_{S}}, \\
L_{\max } & \triangleq \max \left(L_{S D}, L_{S R D}\right), \text { and } L_{S R D}=L_{S R}+L_{R D}-1,
\end{aligned}\right.
$$

and

$$
\widetilde{\mathbf{H}}_{l}^{(k)}=\left[\begin{array}{ll}
\gamma \sqrt{\mathrm{E}_{S R} \mathrm{E}_{R D}} \underline{\mathbf{H}}_{l}^{S R D^{(k)}} & \sqrt{\mathrm{E}_{S D}} \underline{\mathbf{H}}_{2, l}^{S D^{(k)}}
\end{array}\right]
$$

is a $N_{D} \times 2 N_{S}$ complex matrix. In (10), matrices $\underline{\mathbf{H}}_{l}^{S R D^{(k)}}$ and $\underline{\mathbf{H}}_{i, l}^{S D^{(k)}}$ for $i \in\{1,2\}$ are given by

$$
\left\{\begin{array}{llc}
\underline{\mathbf{H}}_{i, l}^{S D^{(k)}} & =\mathbf{H}_{i, l}^{S D^{(k)}}, & 0 \leq l \leq L_{S D}-1 \\
\underline{\mathbf{H}}_{i, l}^{S D^{(k)}} & =\mathbf{0}_{N_{D} \times N_{S}}, & l \geq L_{S D} \\
\underline{\mathbf{H}}_{l}^{S R D^{(k)}} & =\mathbf{H}_{l}^{S R D^{(k)}}, & 0 \leq l \leq L_{S R D}-1 \\
\underline{\mathbf{H}}_{l}^{S R D^{(k)}} & =\mathbf{0}_{N_{D} \times N_{S}}, & l \geq L_{S R D}
\end{array},\right.
$$




$$
\mathbf{H}_{l}^{S R D^{(k)}}=\sum_{p=\max \left(0, l-L_{S R D}+1\right)}^{\min \left(l, L_{S R D}-1\right)} \mathbf{H}_{p}^{R D^{(k)}} \mathbf{H}_{l-p}^{S R^{(k)}} .
$$

The additive noise vector $\widetilde{\mathbf{n}}_{D, t}^{2,(k)}$ at the destination side during the second slot is expressed as

$$
\widetilde{\mathbf{n}}_{D, t}^{2,(k)}=\sqrt{\mathrm{E}_{R D}} \sum_{l=0}^{L_{R D}-1} \mathbf{H}_{l}^{R D^{(k)}} \mathbf{n}_{R,(t-l) \bmod T}^{(k)}+\mathbf{n}_{D, t}^{2,(k)} .
$$

Since $\mathbf{n}_{D, t}^{2,(k)}$ has statistical properties similar to those of $\mathbf{n}_{D, t}^{1,(k)}$, the conditional covariance matrix of $\widetilde{\mathbf{n}}_{D, t}^{2,(k)}$ is given by

$$
\begin{aligned}
\Theta_{\widetilde{\mathbf{n}}_{D, t}^{2,(k)}} \mid\left\{\mathbf{H}_{l}^{R D^{(k)}}\right\}= \\
\quad N_{0}\left[\mathbf{I}_{N_{D}}+\frac{\mathrm{E}_{R D}}{N_{S} \mathrm{E}_{S R}+N_{0}} \sum_{l=0}^{L_{R D}-1} \mathbf{H}_{l}^{R D^{(k)}} \mathbf{H}_{l}^{R D^{(k)}}\right] .
\end{aligned}
$$

To ensure spatially and temporally white noise at the destination node $D$, we rather consider the output $\widetilde{\mathbf{y}}_{D, t}^{2,(k)}=\mathbf{F y}_{D, t}^{2,(k)}$ of the whitening filter $\mathbf{F}=\mathbf{L}^{-1}$, where

$$
\Theta_{\widetilde{\mathbf{n}}_{\mathrm{D}, t}^{2,(k)}} \mid\left\{\mathbf{H}_{l}^{\mathrm{RD}(k)}\right\}=N_{0} \mathbf{L} \mathbf{L}^{\mathrm{H}}
$$

corresponds to the Cholesky factorization of $\Theta_{\widetilde{\mathbf{n}}_{D, t}^{2,(k)}} \mid\left\{\mathbf{H}_{l}^{R D^{(k)}}\right\}$. Then, node $D$ builds up the augmented size signal vector $\mathbf{y}_{D, t}^{e q u^{(k)}}$ which corresponds to reception over two consecutive slots as

$$
\mathbf{y}_{D, t}^{e q u^{(k)}}=\left[\begin{array}{c}
\mathbf{y}_{D, t}^{1,(k)} \\
\widetilde{\mathbf{y}}_{D, t}^{2,(k)}
\end{array}\right] \in \mathbb{C}^{2 N_{D}} .
$$

It can be expressed as

$$
\mathbf{y}_{D, t}^{e q u^{(k)}}=\sum_{l=0}^{L_{\max }-1} \mathbf{H}_{l}^{\text {equ}}{ }^{(k)} \mathbf{z}_{(t-l) \bmod T}+\mathbf{n}_{D, t}^{e q u^{(k)}}
$$

where

$$
\mathbf{H}_{l}^{e q u^{(k)}}=\left[\begin{array}{cl}
\sqrt{\mathrm{E}_{S D}} \underline{\mathbf{H}}_{1, l}^{S D^{(k)}} & \mathbf{0}_{N_{D}} \times N_{S} \\
\gamma \sqrt{\mathrm{E}_{S R} \mathrm{E}_{R D}} \mathbf{L}^{-1} \underline{\mathbf{H}}_{l}^{S R D^{(k)}} & \sqrt{\mathrm{E}_{S D}} \mathbf{L}^{-1} \underline{\mathbf{H}}_{2, l}^{S D^{(k)}}
\end{array}\right]_{(18)}
$$

is a $2 N_{D} \times 2 N_{S}$ complex matrix, and

$$
\mathbf{n}_{D, t}^{e q u}{ }^{(k)}=\left[\begin{array}{c}
\mathbf{n}_{D, t}^{1,(k)} \\
\mathrm{L}^{-1} \widetilde{\mathbf{n}}_{D, t}^{2,(k)}
\end{array}\right] \sim \mathcal{N}\left(\mathbf{0}_{2 N_{D} \times 1}, N_{0} \mathbf{I}_{2 N_{D}}\right) .
$$

Signal vector $\mathbf{y}_{D, t}^{e q u^{(k)}}$ is then used jointly with all the previously received signals $\mathbf{y}_{D, t}^{e q u^{(k-1)}}, \cdots, \mathbf{y}_{D, t}^{e q u^{(1)}}$ to decode the data packet. This leads to the $2 N_{D} k \times 2 N_{s}$ block communication model given by

$$
\underbrace{\left[\begin{array}{c}
\mathbf{y}_{D, t}^{e q u^{(1)}} \\
\vdots \\
\mathbf{y}_{D, t}^{e q u^{(k)}}
\end{array}\right]}_{\mathbf{y}_{D, t}^{e q u, k}}=\sum_{l=0}^{L_{\max }-1} \underbrace{\left[\begin{array}{c}
\mathbf{H}_{l}^{e q u^{(1)}} \\
\vdots \\
\mathbf{H}_{l}^{e q u^{(k)}}
\end{array}\right]}_{\mathbf{H}_{l}^{\text {equ }, k}} \mathbf{z}_{(t-l) \bmod T}+\underbrace{\left[\begin{array}{c}
\mathbf{n}_{D, t}^{e q u^{(1)}} \\
\vdots \\
\vdots \\
\mathbf{n}_{D, t}^{e q u^{(k)}}
\end{array}\right]}_{\mathbf{n}_{D, t}^{e q u, k}} .
$$

\section{Relay ARQ with Slots-Mapping Reversal (SMR)}

Instead of transmitting sub-packet $\mathbf{z}_{1}$ followed by $\mathbf{z}_{2}$ during two consecutive time slots for all ARQ rounds, we propose in this version to reverse the sub-packet transmission order at each ARQ round $k$.
Figure 2.b shows that depending on the transmission index parity, subpackets $\mathbf{z}_{1}$ and $\mathbf{z}_{2}$ are mapped (i.e., transmitted) onto either the first or the second time slot. This approach enables a better exploitation of the available relay and ARQ diversities, and therefore leads to a substantial gain in transmission performance. The equivalent block communication model (20) is still valid for the proposed protocol except for the form of matrices $\mathbf{H}_{l}^{e q u^{(k)}}$ which becomes $k$ parity-dependent, i.e.,

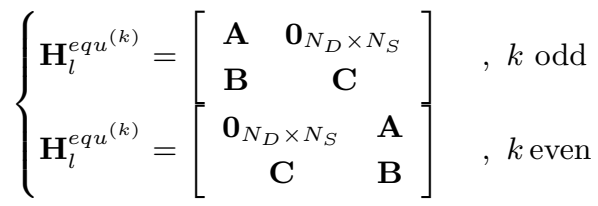

where $\mathbf{A}, \mathbf{B}$, and $\mathbf{C}$ are matrices having the following expressions,

$$
\begin{gathered}
\mathbf{A}=\sqrt{\mathrm{E}_{S D}} \underline{\mathbf{H}}_{1, l}^{S D^{(k)}}, \\
\mathbf{B}=\gamma \sqrt{\mathrm{E}_{S R} \mathrm{E}_{R D}} \mathrm{~L}^{-1} \underline{\mathbf{H}}_{l}^{S R D^{(k)}}, \\
\mathbf{C}=\sqrt{\mathrm{E}_{S D}} \mathrm{~L}^{-1} \underline{\mathbf{H}}_{2, l}^{S D^{(k)}} .
\end{gathered}
$$

\section{Outage Probability And Average Throughrut}

In this section, we investigate both the outage probability and average throughput as two meaningful performance limits of the relay ARQ system under focus.

\section{A. Outage Probability}

Pertaining to (20) for $k=1$, the outage probability at a given signalto-noise ratio (SNR) $\rho$, denoted by $P_{\text {out }}$, refers to the probability half of the information rate $\mathcal{I}$ (the factor $\frac{1}{2}$ comes from the fact that one channel use of the equivalent received signal model (17) corresponds to two temporal channel uses), between transmitted block $\mathbf{z}$ and received block $\underline{\mathbf{y}}_{\mathrm{D}}^{\text {equ,1}}$, is below a target rate $\mathcal{R}$,

$$
P_{\text {out }}(\rho, \mathcal{R})=\operatorname{Pr}\left\{\frac{1}{2} \mathcal{I}\left(\underline{\mathbf{z}}_{\mathbf{y}_{\mathrm{D}}^{e q u, 1}}^{\text {qu }} \mid\left\{\mathbf{H}_{l}^{\text {equ, }}\right\}, \rho\right)<\mathcal{R}\right\}
$$

where

$$
\underline{\mathbf{z}}=\left[\begin{array}{c}
\mathbf{z}_{1} \\
\vdots \\
\mathbf{z}_{\frac{T}{2}}
\end{array}\right] \text {, and } \underline{\mathbf{y}}_{\mathrm{D}}^{e q u, 1}=\left[\begin{array}{c}
\mathbf{y}_{\mathrm{D}, 1}^{e q u, 1} \\
\vdots \\
\mathbf{y}_{\mathrm{D}, \frac{T}{2}-1}^{e q u, 1}
\end{array}\right]
$$

The equivalent virtual MIMO channel $\left\{\mathbf{H}_{l}^{e q u, 1}\right\}_{0 \leq l \leq L_{e q u}-1}$ is assumed quasi-static and unknown to the transmitting nodes $S$ and $R$. Furthermore, it may independently change from round to round referring to as a short-term static channels. It is a contrived assumption, as most papers consider, to choose Gaussian channel inputs, i.e., elements of $\underline{\mathbf{z}}$ follow a Gaussian distribution, for calculating the mutual information rate $\mathcal{I}$. Rather, the more realistic one consists of constraining the inputs to be from a specific constellation alphabet. The only weakness of this method is the computational complexity required to evaluate the constrained information rate. It increases dramatically as $\mathcal{O}\left(4^{M N_{S}\left(L_{\max }-1\right)}\right)$ for high-order constellations and large MIMO systems with moderate-to-large channel memories, where $M$ is the constellation order. Therefore, we resort to the first assumption as an alternative that in any way still gives a valid approximation of the real outage probability especially for high loaded transmission scenarios [17], [18]. Information rates for frequency selective fading MIMO channels have been addressed in [19], [20]. To extend these results toward expressing the outage probability for our ARQ relay system, we use the renewal theory [21] as well as the observation that allows us to view the presented Chase-type ARQ mechanism, with a maximum 


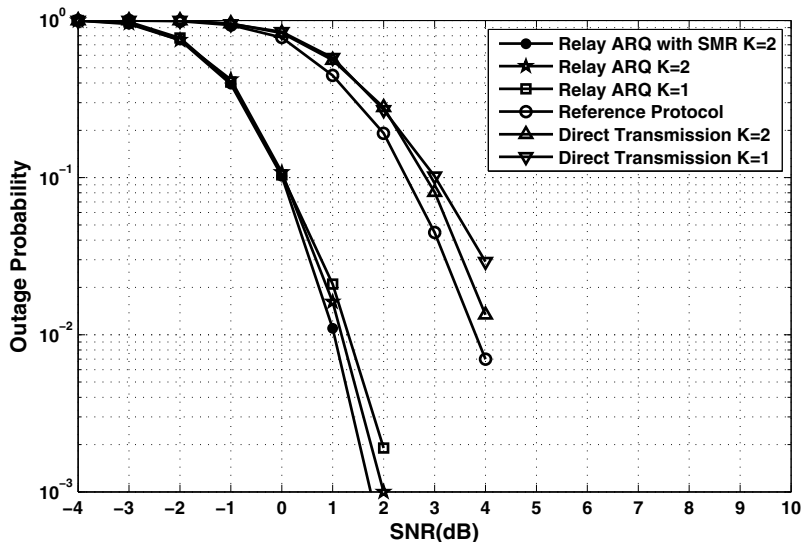

Figure 3. Outage probability versus SNR for $l_{S R}=0.3, N_{S}=N_{R}=N_{D}=$ $2, L_{S R}=L_{R D}=L_{S D}=3$, and $\kappa=3$.

number of rounds $K$, as a repetition coding scheme over $K$ parallel sub-virtual channels [22, p. 194]. Accordingly, given the equivalent MIMO-ARQ channel model (20), (25) can be re-written as

$$
\begin{aligned}
P_{\text {out }}(\rho, \mathcal{R})=\operatorname{Pr}\{ & \frac{1}{2 K} \mathcal{I}\left(\underline{\mathrm{z}} ; \underline{\mathbf{y}}_{\mathrm{D}}^{\text {equ }}, K \mid\left\{\mathbf{H}_{l}^{\text {equ }, K}\right\}, \rho\right)<\mathcal{R}, \\
& \left.\overline{\mathcal{A}}_{1}, \ldots, \overline{\mathcal{A}}_{K-1}\right\}
\end{aligned}
$$

where $\overline{\mathcal{A}}_{k}$ represents the event that a NACK feedback is sent to the source node $S$ at round $k=1, \ldots, K-1$. Though it is quiet complicated to obtain the analytical expression of the outage probability, in the following section we carry out Monte-Carlo simulations to estimate (26).

\section{B. Average Throughput}

It follows from [23] that the average throughput formula corresponding to the transmission over the equivalent MIMO-ARQ channel (18) is given by

$$
\eta=\frac{\mathrm{E}[\mathrm{R}]}{\mathrm{E}[\nu]},
$$

where $\mathrm{R}$ is a discrete random variable (RV) which is equal either to $\mathcal{R}$ when successful packet decoding is detected within the $K$ rounds or 0 otherwise. In an outage sense, these two values are taken with probabilities $1-P_{\overline{\text { out }}}(\rho, \mathcal{R})$ and $P_{\overline{\text { out }}}(\rho, \mathcal{R})$, respectively. $\nu$ is a RV counting the number of rounds consumed to transmit one packet. Thus, the average throughput (27) can be re-expressed as

$$
\eta=\mathcal{R}_{\nu}\left(1-P_{\overline{\text { out }}}(\rho, \mathcal{R})\right)
$$

where $\mathcal{R}_{\nu}=\mathcal{R} / \mathrm{E}[\nu]$. Note that the outage probability $P_{\overline{\text { out }}}$, unlike $P_{\text {out }}$, does not capture the rate distortion effect caused by multiple retransmissions since we do not divide the mutual information rate $\mathcal{I}$ by $K$ similarly to (26), but obviously the entire throughput $\eta$ is influenced by this effect through $\mathrm{E}[\nu]$.

\section{Simulation Results}

Monte-Carlo simulations are conducted to evaluate the outage probability (26) and average throughput (27) performances. Our main purpose in this section is to show that the proposed protocol offers high outage probability and throughput performance compared with the so-called Protocol II [24] where the source broadcasts to both

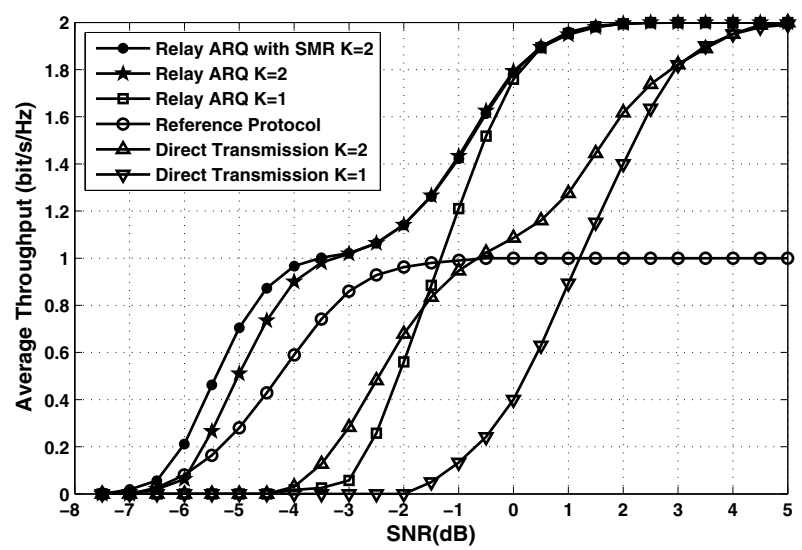

Figure 4. Average throughput versus SNR for $l_{S R}=0.3, N_{S}=N_{R}=$ $N_{D}=2, L_{S R}=L_{R D}=L_{S D}=3$, and $\kappa=3$.

the relay and the destination during the first slot while only the relay node transmits to the destination during the second slot. Therefore, for the sake of fair comparison, we assume a maximum number of ARQ rounds $K=2$ (since Protocol II requires two rounds per each symbol packet transmission). We also provide performance of conventional MIMO-ARQ transmission where relaying is not used. In all figures, Protocol II is referred to as "reference protocol".

All nodes have the same number of antennas, $N_{S}=N_{R}=N_{D}=$ 2. Channel (1), channel (2), and channel (3) have the same length i.e., $L_{S D}=L_{S R}=L_{R D}=3$, with equal power profiles, but unbalanced in terms of shadowing and path loss effect. Without loss of generality, we consider a normalized configuration in which the relay node $R$ is located between node $S$ and node $D$ such that $l_{S R}+l_{R D}=l_{S D}=1$, where $l_{A B}$ denotes the distance between node $A \in\{S, R\}$ and node $B \in\{R, D\}$. Channel energy $E_{A B}$ is also considered to be $E_{A B}=$ $l_{A B}^{-\kappa}$ where $\kappa$ is the parameter characterizing the path loss exponent. The SNR in all figures corresponds to that of the direct link and is defined as

$$
\mathrm{SNR}=\frac{E_{S D} E_{b}}{N_{0}},
$$

where $E_{b}$ presents, in the absence of the relay node $R$, the received energy at node $D$ per useful bit per receive antenna. In the following, we focus on two particular scenarios where the relay node is either close to the source $S$ or the destination $D$.

1) Scenario 1: Relay close to Source $\left(l_{S R}=0.3\right)$ : Figure 3 shows that both relay ARQ communication schemes (i.e., with and without SMR) provide superior outage probability performance in comparison to Protocol II. The SNR gap is about $2.5 \mathrm{~dB}$ at $10^{-1}$ outage probability. More interestingly, both mapping techniques achieve high SNR gains starting from the first round due to relay diversity captured by the proposed sub-packet transmission strategy illustrated in Fig. 2. Also, note that the proposed technique outperforms Protocol II in terms of average throughput as it can be seen from Fig. 4. While the latter suffers from the half-duplex constraint leading to throughput saturation at $1 \mathrm{bit} / \mathrm{s} / \mathrm{Hz}$ for $\mathrm{SNR}$ values greater than $-1.5 \mathrm{~dB}$, relay ARQ achieves this throughput value at only $-3.5 \mathrm{~dB}$ SNR.

2) Scenario 2: Relay close to Destination $\left(l_{S R}=0.7\right)$ : From Fig. 5 , we see that relay ARQ with SMR provides a relay diversity gain about $2 \mathrm{~dB}$ along with an ARQ gain of about $0.7 \mathrm{~dB}$ that appear at the first and the second rounds, respectively. Thus, a sum of $2.7 \mathrm{~dB}$ at $10^{-2}$ outage probability is attained. The relay node location is therefore a determinant factor that greatly influences these gains. 


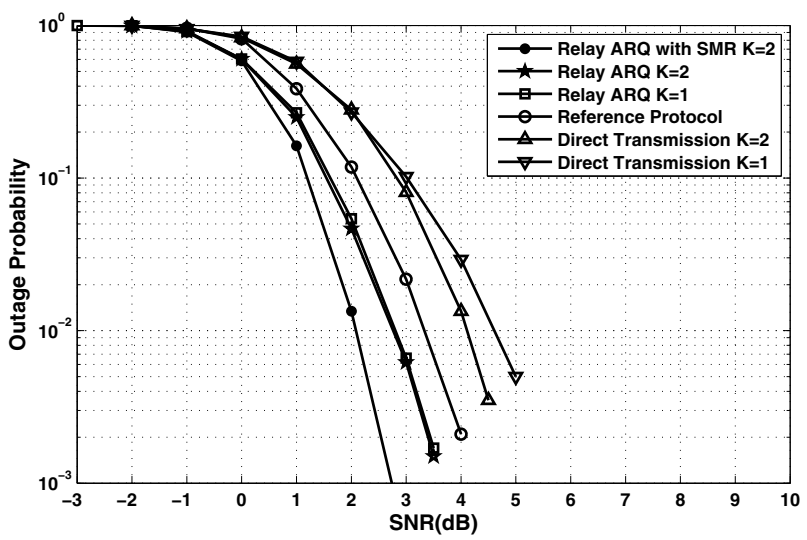

Figure 5. Outage probability versus SNR for $l_{S R}=0.7, N_{S}=N_{R}=$ $N_{D}=2, L_{S R}=L_{R D}=L_{S D}=3$, and $\kappa=3$.

Average throughput curves are reported in Fig. 6. We observe that the proposed relay ARQ protocol without SMR suffers from throughput loss due to the long distance between the source and the relay. Conventional relaying provides better throughput performance for the region of low SNR values (typically for SNR $<-2 \mathrm{~dB}$ ). Surprisingly, relay ARQ with SMR achieves better throughput performance compared with conventional relaying for the entire SNR region. This improvement is due to the fact that the destination node $D$ leverages the ARQ gain at the second round $K=2$ in enhancing the average throughput.

\section{CONCLUSION}

In this paper, we investigated throughput-efficient relay ARQ techniques for cooperative single carrier transmission over broadband MIMO channels. We introduced two new protocols where only one time slot duration is required for transmitting one data packet at each ARQ round. We evaluated the average throughput and outage error probability performance and showed that the proposed technique significantly reduces the multiplexing loss due to the half-duplex constraint while providing attractive outage error probability performance.

\section{REFERENCES}

[1] J. N. Laneman, G. W. Wornell, and D. N. C. Tse, "An efficient protocol for realizing cooperative diversity in wireless networks," in Proc. IEEE Intern. Symp. Info. Theory (ISIT), Washington, DC, Jun. 2001.

[2] A. Sendonaris, E. Erkip, and B. Aazhang, "User cooperation diversity Part I \& Part II," IEEE Trans. Commun., vol. 51, pp. 1927-1948, Nov. 2003.

[3] E. Zimmermann, P. Herhold, and G. Fettweis, "The impact of cooperation on diversity-exploiting protocols," in Proc. IEEE Veh. Techn. Conf. (VTC), Milan, Italy, May 2004

[4] K. Azarian, H. El Gamal, and P. Schniter, "On the achievable diversitymultiplexing trade-off in half-duplex cooperative channels," Trans. Info. Theory, vol. 51, pp. 4152-4172, Dec. 2005.

[5] J. Boyer, D. D. Falconer, and H. Yanikomeroglu, "Multihop diversity in wireless relaying channels," IEEE Trans. Commun., vol. 52, pp. 18201830, Oct. 2004.

[6] J. N. Laneman, D. Tse, and G. W. Wornell, "Cooperative diversity in wireless networks: Efficient protocols and outage behavior," IEEE Trans. Info. Theory, vol. 50, no. 12, pp. 3062-3080, Dec. 2004.

[7] G. Yu, Z. Zhang, and P. Qiu, "Cooperative ARQ in wireless networks: Protocols description and performance analysis," in Proc. IEEE Intern. Conf. Commun. (ICC), Istanbul, Turkey, June 2006.

[8] F. Atay Onat, H. Yanikomeroglu, and S. Periyalwar, "Relay-assisted spatial multiplexing in wireless fixed relay networks," in Proc. IEEE GLOBECOM, San Francisco, USA, Nov.- Dec. 2006.

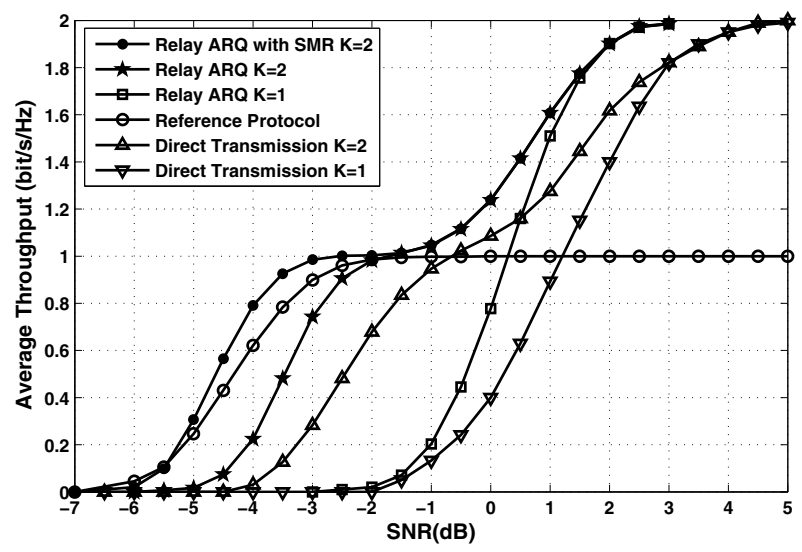

Figure 6. Average throughput versus SNR for $l_{S R}=0.7, N_{S}=N_{R}=$ $N_{D}=2, L_{S R}=L_{R D}=L_{S D}=3$, and $\kappa=3$.

[9] H. Chafnaji, T. Ait-Idir, H. Yanikomeroglu, and S. Saoudi, "On the design of turbo packet combining schemes for relay-assisted systems over multiantenna broadband channels," in Proc. IEEE Veh. Techn. Conf. (VTC), Taipei, Taiwan, May 2010.

[10] Y. Fan, C. Wang, J. Thompson and H.V. Poor, "Recovering multiplexing loss through successive relaying using repetition coding," IEEE Trans. Wireless Commun., vol. 6, no. 12, pp. 4484 - 4493, Dec. 2007.

[11] R. Tannious and A. Nosratinia, "Spectrally efficient relay selection with limited feedback," IEEE Journal Select. Areas Commun., vol. 26, no. 8, pp. 1419-1428, Oct. 2008.

[12] R. Narasimhan, "Throughput-delay performance of half-duplex hybridARQ relay channels," in Proc. IEEE Intern. Conf. Commun. (ICC), Beijing, China, May 2008.

[13] T. Tabet, S. Dusad, and R. Knopp, "Achievable diversity-multiplexingdelay trade-off in half-duplex ARQ relay channels," in Proc. IEEE ISIT, Adelaide, Australia, Sept. 2005.

[14] Y. Qi, R. Hoshyar, and R. Tafazolli, "On the performance of HARQ with hybrid relaying schemes," in Proc. IEEE Intern. Conf. Commun. (ICC), Dresden, Germany, Jun. 2009.

[15] H. Mheidat, M. Uysal, and N. Al-Dhahir, "Equalization techniques for distributed space-time block codes with amplify-and-forward relaying," IEEE Trans. Signal Processing, vol. 55, pp. 1839-1852, May 2007.

[16] H. Xiong and J. X. P. Wang, "Frequency-domain equalization and diversity combining for demodulate-and-forward cooperative systems," in Proc. IEEE ICASSP, Las Vegas, NV, Mar.-Apr. 2008.

[17] T. Ait-Idir and S. Saoudi, "Turbo packet combining strategies for the MIMO-ISI ARQ channel," IEEE Trans. Commun., vol. 57 , no. 12, pp. 3782-3793, Dec. 2009.

[18] R.Visoz, A.O. Berthet, and S. Chtourou, "A new class of iterative equalizers for space-time BICM over MIMO block fading ISI AWGN channel," IEEE Trans. Commun, vol. 53, no. 12, pp. 2076-2091, Dec. 2005.

[19] H. El Gamal, A. R. Hammons, Y. Liu, M. P. Fitz, and O. Y. Takeshita, "On the design of space-time and space-frequency codes for MIMO frequencyselective fading channels," IEEE Trans. Info. Theory, vol. 49, no. 9, pp. 2277-2292, Sep. 2003.

[20] Z. Zhang, T. M. Duman, and E. M. Kurtas, "Achievable information rates and coding for MIMO systems over ISI channels and frequency-selective fading channels," IEEE Trans. Commun., vol. 52, no. 10, pp. 1698-1710, Oct. 2004.

[21] R. Wolff, Stochastic Modeling and the Theory of Queues, Upper Saddle River, NJ: Prentice-Hall, 1989.

[22] D. Tse and P. Viswanath, Fundamentals of Wireless Communication, Cambridge University Press, May 2005.

[23] G. Caire and D. Tuninetti, "The throughput of hybrid-ARQ protocols for the Gaussian collision channel," IEEE Trans. Info. Theory, vol. 47, no. 5, pp. 1971-1988, July 2001.

[24] R. U. Nabar, H. Bolcskei, and F. W. Kneubuhler, "Fading relay channels: performance limits and space-time signal design," IEEE Journal Select. Areas in Commun., vol. 22, no. 6, pp. 1099-1109, Aug. 2004. 